\title{
Ludi Lodovico \\ Wem das Posthorn bläst - zur Konstruktion und Konjunktur des Nationalen
}

\section{Einleitung}

Wiederholt sich die Geschichte? 1917/18 - nach der revolutionären bzw. militärischen Beseitigung des zaristischen Reiches, der Donaumonarchie und der letzten Reste des osmanischen Reiches auf dem Balkan - erschien der National«staat als Zauberformel: jedem Kind einen Lampion und jeder *Nation* ihren eigenen Staat. Das deckt sich mit der *Burlesken Träumerei* des unvergleichlichen Erik Satie: "Ich finde, daß alle Franzosen, die auf französischem Gebiet geboren sind, von französischen Eltem oder solchen, die diesen Anschein erwecken, ein Anrecht auf eine Anstellung bei der Pariser Post haben sollten*. Mit der Post hat der moderne Begriff »Nation* immerhin soviel zu tun, daß er von Paris aus seine Reise antrat und daß Lenin außer für »nationale« Selbstbestimmung für die deutsche Post schwärmte.

Wenn man die Reaktionen auf den Untergang der Sowjetunion durchsieht, so scheint es, als hätten die Kommentatoren nicht Post aus Paris, sondern aus Washington erhalten. Haben sie Woodrow Wilson persönlich zum ghost-writer erkoren? »Nationale Selbstbestimmung " und "National«staat, die sich als Volksvorurteile in den Himen festgefressen haben, gelten auf jeden Fall wieder einmal als erstrebenswerter globaler politischer Normalzustand. Die abenteuerliche Prognose, die Völker der ehemaligen Sowjetunion würden jetzt gleichsam mnormalisiert* und in die europäische Tradition des vermeintlich selbstverståndlichen und demokratischen Nationalismus zurïckgeholt, müßte der Redlichkeit halber die Tribute nennen, die die Menschen in Europa seit etwa 200 Jahren entrichtet haben für derlei Normalität.

Wohin man heute zwangsläufig gerät, wenn man noch eimmal versucht, die Welt nach »Nationen $*$, Nationalstaaten $*$ oder der Fiktion des »nationalen Selbstbestimmungsrechts* einzuričnten, führen die politischen und militärischen Eliten im ehemaligen Jugoslawien praktisch vor, und große Teile der deutschen Presse sowie das Stammtischgerede begleiten das Geschehen mit wohlfeilen, also *serbenfresserischen Kommentaren. Allen voran trabt die FAZ. Schon am 12.12.1990 entdeckte J.G. Reißmüller bei den Serben eine Spezialität, die es ihm so angetan hat, daß er nun bald 
täglich darauf zurückkommt: »die uneuropäische Politik des Unterdrükkens anderer Völker*. Im Baltikum genauso: »die ganze Unterdrückungsaktion in Litauen ist uneuropåisch * bzw. "asiatisch», vulgo: »sowjetisch * (15.1.1991). Wem das alles irgendwie bekannt vorkommt, ist auf der richtigen Spur. Was authentisch europåisch ist, hat Treitschke längst beschrieben: „Wenn die Engländer ... die Hindus vor die Mündungen der Kanonen banden und sie 'zerbliesen', daf ihre Körper in alle Winde zerstoben, so kann man das, da doch der Tod sofort eintrat, nicht tadeln. $\mathrm{Daß}$ in solcher Lage Mittel des Schreckens angewendet werden müssen, ist klar $\%$ Bei Reißmüller ist auch alles klar - im Namen des »nationalen Selbstbestimmungsrechts* klagt er die nationale "Anerkennung* ein, deren Verweigerung ebenso unterhalb des *europåischen Standards liegen soll wie die Skepsis gegenüber dem nnationalen Selbstbestimmungsrecht*, das nun als Krone der »europäischen Rechtskultur* (FAZ 17.7.91) herhalten muß, - Man kann sich theoretisch fragen, im Namen wellcher Prinzipien und Ideologien in den letzten 200 Jahren am gründlichsten gemordet wurde, oder man kann ganz empirisch die Friedhöfe der Geschichte des 19. und 20. Jahrhunderts besichtigen - eines steht außer jedem Zweifel: der Politik und Staatsbildung nach "nationalen* Kriterien, dem Popanz $»$ Nationalstaat« also, kann niemand streitig machen, daß er vom jugendlichen, revolutionären und zeitweise emanzipatorischen Heroen zum bloßen Zulieferer der Totengräber verkam. Nationalstaat" und »nationale« Selbstbestimmungsrhetorik veralteten schnell, was unmittelbar mit der Erzeugung, Fraxis und Rechtfertigung von Nationen*, *Nationalitäten* und *Nationalstaaten* zu tun hat. Die vermeintliche Krone meuropåischer Rechtskultur* lebt von theoretischen Improvisationen und billigen Ideologemen; "nationale; Selbstbestimmung ist heute substanzlose Gesinnungshuberei oder, wenn sie ernsthaft praktiziert wird, eine Kannibalen-Parole.

\section{Nation - in jeder Hinsicht ein Gemachtes}

Mangels tragfähiger Grundlagen in der Gegenwart wichen die Ideologen des $\gg$ Nationalstaats* immer auf die Geschichte aus. Die fehlende Kohärenz des Prinzips sollte sich angeblich aus menschlicher Natur, gemeinsamer Abstammung, gemeinsamer Sprache, Geschichte und Kultur ergeben. Schon bei oberflächlicher Betrachtung erweist sich jedoch das Nationale als ein in langen und komplizierten Prozessen entstandenes historisch-politisches Konstrukt und nicht als quasi-natürliche Gegebenheit. Der im 19. Jahrhundert im Windschatten der nationalen Bewegungen mer- 
fundene* Syllogismus, wonach sich ein allemal »tatenhungriges* Volk (1) im Krieg zur Nation formiert (2) und dabei die »fremden* Bevölkerungsteile to̊tet, vertreibt, unterwirft oder assimiliert, um schließlich nach langem Ringen den angeblichen $*$ Normalzustand*, d.h. den Status eines ethnisch homogenen $»$ Nationalstaats zu erreichen (3), ist eine nationalistische Legende oder, was schlimmer ist: eine Rechtfertigung des Hinmordens Unzähliger zur höheren Ehre von *Nation*, "Nationalstaat* und "Vaterland«. Solche historischen Legenden drechseln das Nationale zum Subjekt der Geschichte, so als ob das angeblich in allen Völkern schlummernde Nationale von Anfang an nur darauf gewartet hätte, vom Siegfried $»$ Nationalstaat wachgeküßr zu werden, um die Nicht-Nationalen zu vertreiben, zu schlachten oder zu assimilieren. Je später das Wachküssen der Nation faktisch stattfand, desto tiefer wurde »das Nationale in den Seelen der Individuen und Völker verankert - exemplarisch durch die deutsche Geschichtswissenschaft. Im Bemühen, die Zeugung der Nation möglichst weit nach hinten zu verlegen, um die Spätgeburt zu »erklären* und gleichzeitig aus dem Alter jener Urkopulation der zu spät und zu kurz gekommenen "Nation" der Gegenwart Geltungsansprüche, Vorrechte, Ưberlegenheit und dergleichen zuzuschlagen, haben Generationen von Historikern Bibliotheken zusammengeschrieben.

Bei Licht besehen ein Gebirge von papierner Vergeblichkeit, denn für die Begriffe Volk und Nation sieht die Bilanz in der kritischen Forschung trübe aus. Für kein einziges Großvolk kann man ein hohes Alter, ungebrochene Kontinuität oder gar - liebstes Kind der Nationensucher und Nationalitätenbastler - ethnische Homogenităt belegen. Und was die Anfänge, Wiegen und Geburtsstunden von Großvölkem und Nationen betrifft, so liegen sie nicht im geheimnisvollen mythologischen Dunkel, im Kyffhäuser, auf den Schlachtfeldern vor Troja oder im Frühmittelalter, sondern ganz profan zwischen Buchdeckeln bzw。 auf der platten Hand: "In einem gewissen Sinne sind es die Historiker, die die Nationen schaffen* (Guenée 1971, S.123).

Verkürzt und vereinfacht (Vgl. Wenskus 1961, S.82f): am Anfang stand nirgendwo ein großes Volk und schon gar nicht eine ethnisch oder sprachlich homogene Nation, sondern eine Vielfalt von $*$ bunten * gentilen Verbånden. Diese Verbände Ståmme zu nennen, verbietet die Tatsache, daß mit dem Begriff "Stamm immer noch die legendäre gemeinsame $A b$ stammung, Sprache und Kultur verbunden wird. Genau das war jedoch nicht der Fall. Die gentile Entwicklungsphase ist überall gekennzeichnet durch eine Vielfalt von Siedlungsformen, inneren sozialen Beziehungen, Herrschaftsverhåltnissen und ethnischer Mischungsverhältnisse. Erst relativ spåt bilden sich gentile Verbånde zu Vo̊lkern und Großvölkem, wo- 
bei deren einzelne Bestandteile in Sprache, Kultur und Sitten sehr lange resistent bleiben gegenüber den vereinheitlichenden (»Nationalisierungs- $«$ ) Tendenzen.

So ist es z.B. nichts als ein Gerïcht, die Franken seien das Urvolk und die Begründer "Frankreichs«. Der Anteil eingewanderter (!) Franken am bunten Völkergemisch aus Römern, Galliem, Kelten, Bretonen, Normannen, Burgundern etc., aus dem zwischen Mittelalter und Neuzeit, von dem kleinen Gebiet der Ile de France ausgehend, Frankreich heranwächst, ist minimal. Einzig im Seinebecken dürfte der Anteil der Franken im 6./7.Jh. um zehn Prozent betragen haben, sonst überall bedeutend weniger (Werner 1984, S.358). Nicht die Franken ethnischer Herkunft bilden Frankreich, sondern Herrschergeschlechtern und sozialen Eliten mit zum Teil fränkischen Vorfahren ist es im Laufe der Jahrhunderte gelungen, die anderen in $*$ Frankreich* siedelnden, einwandernden und sich vermischenden gentilen Verbånde, Völkerschaften und "Stämme* sowie deren herrschende Schichten zu einem Gemeinwesen zu formen. "Unterwerfung gegen Schutz* vor inneren und äußeren Feinden lautete die Formel, nach der im Laufe der Jahrhunderte so etwas wie ein Staat im modernen Sinne und *nationaler* Zusammenhalt geschaffen wurden; dieser "nationale* Zusammenhalt umfaßte jedoch in Frankreich bis zur Revolution explizit nur die oberen Stände. Von der nachrevolutionären Nation unterscheidet sich dieser Begriff grundsätzlich, obwohl ihm dasselbe Wort zugrunde liegt. Das einfache Volk dagegen blieb, was es war: normannische, aquitanische, gaskognische Magd, Bäuerin oder Handwerkersfrau; provenzalischer, bretonischer, burgundischer etc. Bauer, Knecht oder Handwerker; und genauso redeten sie vielerlei Sprachen, nur nicht Französisch. *Frankreich* erschien dem menu peuple noch bis ins 18. Jahrhundert hinein als ein ebenso unverständliches und künstliches Produkt wie die dazugehörige Hochsprache Französisch: eine Sache der weltlichen und kirchlichen Herren, mit der das gemeine Volk nichts zu schaffen hatte. Bei den anderen europäischen Großvölkern und Nationen verhielt es sich nicht anders.

Ludwig XIV.: $\rightsquigarrow$ Die Nation ist kein Staatsstand (corps d'État) in Frankreich ${ }$, und $»$ die Nation ist vollständig in der Person des Königs verkörpert». Keine hundert Jahre später wird Emmanuel Joseph Sieyes seinen fulminanten Traktat mit dem Satz beginnen: $*$ Der dritte Stand ist eine vollständige Nation* (Sieyes 1789, S.121).1 Das war keine empirische Beschreibung, sondern ein bürgerlich-revolutionäres Programm - in sei-

1 Im intellektuellen Horizont eines FAZ-Leitartikels erscheint Frankreich« trotzdem mit wseiner tausendjährigen Geschichte als stolzer Nationalstaak (FAZ v. 21.4.92, T. v. Münchausen). 
ner ganzen Widersprüchlichkeit zwischen Emanzipation und erneuter Ausgrenzung derer, die man zur Nation nicht, nicht mehr oder noch nicht zählen mochte. Die Französische Revolution schuf den modernen, der Tendenz nach demokratischen Nationsbegriff. Die Beziehung zwischen moderner Nation und moderner Demokratie ist zwar genetisch unbestreitbar, aber praktisch lose und extrem provisorisch, theoretisch irrelevant und juristisch kriminell.

\section{Anachronistische Rückprojektionen: "Deutschland}

Es ist ein fast unauflösbares Volksvorurteil und ein Anachronismus, im Blick auf Frankreich von einer *alten* Nation zu reden, obwohl sich diese über fünf Jahrhunderte langsam und mit Rückschlägen entwickelte und erst in und nach der Revolution von 1789 , vor allem in den Kriegen gegen das aristokratisch-monarchische Europa regelrecht geschaffen wurde. Carnot, der Kriegsminister, gilt nicht nur als »organisateur de la victoire*, sondern auch als einer der Schmiede der modernen Nation, als deren Kinderstube Schule und Kaserne fungieren (»die Schule wird zum Vorraum der Kaserne" Taine 1893, S.229).2 Das ist nicht falsch, greift aber zu kurz, denn es dauerte noch eine ganze Weile, bis der französische Zentralstaat mit seinen Präfekten, Offizieren und Schulmeistern als nationalen Rasenmähern die überkommenen sozialen, kulturellen und sprachlichen Gewächse einheitlich zurechtgestutzt und zur Grande Nation (uni-)formiert hatte. Dazu mußten die Volkssprachen und Dialekte systematisch herabgesetzt werden, bis in der Öffentlichkeit nur noch das akademisch normierte Hauptstadtidiom Französisch aufzutreten wagte; dazu mußten vor allem die allgemeine Schulpflicht und mit ihr eine verbindliche Nationalsprache »eingeführt* werden (»eingeführt* verharmlost die Brutalität, mit der der Pariser Zentralismus regionale Sprachen und Dialekte buch-

2 Die rigorose Sprachpolitik aller französischen Staatsverwaltungen vor und vor allem seit der Revolution bewunderte jenseits des Rheins der bedeutende Romanist K.Vossler: $\star$ Nicht einmal beim Sprechen, geschweige denn beim Schreiben, erlaubt sich der gebildete Franzose volkstümliche Nachläßigkeiten« (Vossler 1913, S.372). Henri IV. sieht seine Muttersprache als $*$ naiven Übergriff« bzw. *Gascognismus« verspottet (ebd.), Flaubert wird professoral schwer zensuriert wegen seiner Neigung zu *Normandismen«.- Als François I. am 15.8.1539 dekretierte, weder Latein noch die Volkssprachen in amtlichen Dokumenten zu verwenden, stieß er auf harten Widerstand (ebd., S.251). Kein Wunder - noch 1789 hätte nur eine Minderheit das Französische als Mutter- oder gar als Umgangssprache bezeichnet. Die Nationalsprache Deutsch hat ihre Wurzeln denn auch nicht bei einem ominösen deutschen Volk«, sondern in der Hofkanzlei Karls IV. in Prag und an den Schreibpulten der Theologen und Professoren zwischen Reformation und Aufklärung. 
stäblich ausrottete).

Es ist schon ein schwieriges Geschäft, nachzuzeichnen, wie die französische Nation in Schule und Kaserne herangezüchtet, von national eingestellten Intellektuellen herbeigeschrieben und wie die französische Hochsprache staatlich verordnet wurde; für das Deutsche ist das noch etwas dornenreicher, weil man es nicht nur mit nationalistisch eingefärbten Anachronismen zu tun hat, sondern seit Beginn der deutschen Geschichtswissenschaft mit einer militanten Ideologieproduktion, die sich - pragmatisch entschärft - in Teilen der deutschen Mediaevistik und Rechtsgeschichte bis heute durchhält.

Der Beginn der deutschen Historiographie fällt zeitlich zusammen mit der Wahrnehmung nationaler, nationalstaatlicher Defizite in den Staaten des Deutschen Bundes gegenüber dem nachrevolutionåren Frankreich. Das sollte kompensiert werden mit einer programmatisch deutschnationalen Lesart der mittelalterlichen Quellen: die Probleme der Gegenwart bestimmten die Wahrnehumng und Aufbereitung der Vergangenheit.

Der *deutschen Nation*, einer geschichtsphilosophischen und politischen Konstruktion par excellence, verliehen national gesinnte Historiker den Charakter eines Naturtatbestandes, indem sie »Germanen* und »Deutsche* nicht nur gleich-, sondern als "von Anfang an* vorhandenes, homogenes Großvolk voraussetzten, das im mittelalterlichen Kaiserreich eine wdeutsche Einheit « angeblich verwirklicht hatte und dann fahrlässig (»Italienpolitik«) verspielte. Die richtige Voraussetzung, daß gentile Verbände, Völkerteile, Völkerschaften, *Stämme und Völker ålter sind als mStaaten «, "Reiche* und "Nationen* verlängerte die deutsche Historiographie und Rechtsgeschichte zur Vorstellung eines immer schon existierenden »deutschen Volkes*, das eben nur Pech gehabt habe mit seinen von Italien berauschten Herrschern und herrschsüchtigen Päpsten und deshalb in punkto Staatlichkeit und Nationalität gegenüber $*$ Frankreich» (das es damals so wenig gab wie ein »deutsches* Kaiserreich) ins Hintertreffen geraten sei.

»Germania« und $*$ Gallia ${ }^{3}$ waren von der ro̊mischen Antike bis ins Mittel-

3 Vgl. zum folgenden Werner (1986, \$.782ff) mit weiterer Literatur. - Daß der zuweilen an nationalem Priapismus leidende Journalist J.Busche $(1991, \mathrm{S.105} \mathrm{ff}$ ) aus dem Ursprung von theodiscus (wlateinischer Neologismusu) die scharfe Vermutung preßt, eben

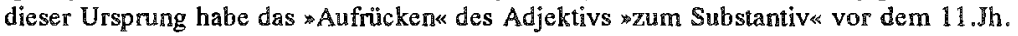
verhindert und nicht die schlichte Tatsache, daß es vor dem 11. Jh. eben riemanden gab, der sich selbst oder andere als $*$ Deutsche betrachtete (ganz zu schweigen von *deutscher Nation", das erst im 14.115.Jh. erscheint und in der Regel nur die Titulatur eines supranationalen Verbandes meint) liegt auf der Hand. Wo jedoch ausgewiesene Fachleute aus der Mediaevistik - gegen die Quellen und gegen den Forschungsstand mehr oder weniger gleich argumentieren, regient der über die Nazi-Zeit hinweg gerettete nationale Obskurantismus: $* \mathrm{Da} B$ der Kaisergedanke und der Begriff des Imperiums 
alter und die Neuzeit hinein geographische Begriffe. Der Rhein bildete die Ost-West-Grenze. Das Wort *Germania* in mittelalterlichen Quellen zielt immer auf diese geographischen Grenzen und nicht auf die ethnische Abstammung der Bevölkerungen oder politische Substrate. Die nach Gallien gewanderten Franken waren ebenso »Germanen* wie die dieseseits des Rheins verbliebenen. Als Deutsche bezeichneten sich freilich weder die einen noch die andern.

Anders verhält es sich mit der Sprachenbezeichnung; »deutsch *theodiscus*, gebildet aus althochdeutsch diot (Volk), meint alle nicht-romanischen Volkssprachen. Die nationes theodiscae umfassen z.B. nichtromanisch sprechende Franken in den heutigen Ländern Flandern, Deutschland und Frankreich, Sachsen in England, Goten und Langobarden in Italien; hier heißen Franken, die nicht-romanisch reden, deshalb teutonici oder tedeschi, und umgekehrt nennen diese die romanische Sprachen verwendende Umgebung *Welsche*. Nicht-romanisch sprechende Sachsen in England oder Franken in Flandern bzw. Italien tauchen in den Quellen als nationes theodiscae auf, können jedoch nicht *deutsche Stämme* oder gar *Deutsche* gewesen sein, da sie niemals dem politischen Gemeinwesen angehörten angehörten, das - 962 als ostfränkisches Reich gegründet erst im 11.Jh. gelegentlich als regnum Teutonicum (1073) auftaucht. Die an-deren Franken, Sachsen usw. gehören selbstverständlich zu den konstituierenden Bestandteilen der anderen im Entstehen begriffenen Großvölker in *England* bzw. "Frankreich*。

Mit "regnum" meinen die mittelalterlichen Quellen einen sekundären Herrschaftsverband; man erklärt sich dessen Entstehung aus seßhaften gentilen Verbänden, die sich eben durch diese Seßhaftigkeit kulturelle, rechtliche und politische Strukturen schaffen und dabei die zu verschiedenen Zeiten eingewanderten wie die »eingeborenen* gentilen Verbånde assimilieren. Diese Teilreiche (regna oder auch patriae) umfaßten also immer Verbände unterschiedlicher Herkunft und Abstammung: Gothia, Burgundia, Francia, Saxonia, Lotharingia, Baioaria, Alemannia, Italia, Provincia sind keine homogenen Gebilde wie die anachronistischen Begriffe *Stamm* oder *Stammesherzogtum* suggerieren, sondern über lange Zeit entstandene melting pots. Die deutsche Uึbersetzung für »regnum* ist in der Regel *rich* oder *lant* (*regnum Baioariae*, *Baiemlant*). Für eine Mehrzahl von regna auf dem Territorium des ostfränkischen Reiches existiert vor dem 16.Jh. für »Deutschland* ganz selbstverständlich und $\gg$ lo- 
gisch* nur der Plural *deutsche Lande*. 4 Der Alemanne, Sachse oder Baier existierte nicht von Anfang an, sondern entstand in einem geschichtlichen Prozeß im Zuge der Vermischung und Verbindung von gentilen Verbänden zu Teilreichen/regna; gleichzeitig als Baier und Deutscher oder Sachse und Deutscher konnte man erst ab dem 11.Jh. bezeichnet werden - gut 500 Jahre nachdem Alemannen, Baiern, Sachsen, Franken usw. belegt sind. Wie unwichtig das "Deutschsein* neben der faktischen Zugehörigkeit zu einem regionalen Teilreich und seiner Herrschaft (regnum, patria) blieb, beweist die Tatsache, daß der politische Begriff »deutsches Volk* erst nach der Französischen Revolution und in Anlehnung an die revolutionäre Terminologie vom souveränen »peuple français« nachzuweisen ist (Werner 1992).

Das »Deutsche Kaiserreich*, angeblich 962 von Otto dem Großen gegründet, gehört zu den Fiktionen der nationalstaatlich imprägnierten Historiographie des 19. Jahrhunderts, die bis heute in schlechten Schulbüchern und im common sense fortleben.5 Ottos $»$ Imperium Romanum* (erst seit 1157 mit dem Zusatz ${ }$ Sacrum* und erst seit 1442 mit der Präzisierung »nationis germanicaek, also: *Heiliges Römische Reich germanischer (!) Nation* und erst seit dem Kölner Reichsabschied von 26.8.1512 und bis zum Untergang des Reiches (12.7.1806) hieß es offiziell und adjektivisch »Heiliges römisches Reich deutscher (!) Nation $*$ ) war nicht eines der »Deutschen*, womöglich gar der »deutschen Nation* im modernen Sinne, sondern jenes der Franci et Saxones (Franken und Sachsen). Diese beanspruchten die Erbschaft des "(Sacrum) Imperium Romanum* für sich. Franci et Saxones mußte man allerdings geographisch näher bezeichnen, um sie z.B. von den *franzősischen* bzw. *englischen « Sachsen und Franken abzugrenzen - deshalb der spätere Hinweis auf die rechtsrheinisch gelegene Germania im Zusatz wnationis germanicae*, nicht etwa »teutonicaek oder »theodiscae . Näherhin meinte Otto d. Gr. mit den Franci et Saxones, in deren Namen er auftrat, nicht *Völker* im modernen Sinne, sondern den in *Kirche* und "Staat « Macht und Herrschaft ausübenden Adel (zusammengefaßt in der Formel: regnum Francorum et Saxonum). Diese weltliche und kirchliche Elite fungiert in den mittelalterlichen Quellen unter dem Begriff »populus* / Volk*, das die Kirchenoberen

4 Der terminologische Wechsel von den $»$ deutschen Landen $\mathrm{zu} »$ Deutschland» geht im wesentlichen zurück auf den Streit unter den Humanisten über die materiellen und kulturellen Fortschritte in Italien und in der Germania, wie sie Tacitus beschrieben hat. Dazu provozierte zuerst die Schrift des Enea Silvio Piccolomini, De ritu, situ, moribus et conditione Germaniae (1457/58), vgl. See (1970, S.14ff).

5 * Gleichwohl wußten die Deutschen im Reich ihrer mittelalterlichen Kaiser, daß sie deutsch waren« (Busche 1991, S.107). Wann und von wem? Post festum und exklusiv von Busche und seinesgleichen. 
und die Könige wählte bzw. absetzte. Mit *Volk« war also - im Unterschied zum heutigen Sprachgebrauch - auschließlich das Kirche und Staat lenkende Volk, eine dünne Oberschicht aus Kriegern, Adligen, Klerikern und Freien, gemeint. 6

$\mathrm{Zu}$ welchen Konfusionen es in Sachen deutsch und $»$ Nation $*$ trotz dieser relativ einfachen und eindeutigen Zusammenhänge nach wie vor kommt, soll an einem Beispiel gezeigt werden. Der ebenso verdienstvolle wie konservative Sozialhistoriker Werner Conze schrieb drei Jahre vor seinem Tod einen Aufsatz unter dem Titel: $\bowtie$ Deutschland $\star$ und $»$ deutsche Nation* als historische Begriffe (Conze 1983).

Hochideologisierter Ramsch wie der Begriff "Stammesnationen (ebd., 24) erscheint darin, aber kein einziger eindeutiger Beleg für das Titelwort »deutsche Nation $*$. Das ist handwerklich gesehen ein Defizit und faktisch das Eingeständnis, daß es frühe Belege für diesen Begriff in politischen Kontexten nicht gibt, weil man sich noch für sehr lange Zeit zwar als "Deutscher* (d.h. deutschsprachiger Bayer, Sachse etc.) bezeichnen konnte, der Ausdruck »deutsche Nation* aber immer die Verbindung zum "Heiligen Römischen Reich deutscher Nation* herstellte, also gerade modern gesprochen - ein supranationales Gebilde meinte, dem selbstverständlich Ungarn, Kroaten, Norweger, Böhmen etc. mitangehörten. Das handwerkliche Defizit gerät Conze jedoch unter der Hand zum peinlichen Lapsus, wenn er als *Beleg* den Abschied des Augsburger Reichstags vom 25.9.1555 heranzieht. Conze suggeriert, hier stünde bereits *die Teutsch Nation, unser geliebt Vaterland* (ebd., S.28)7 in einem modernen Sinne, d.h. als ein explizit und exklusiv auf ein national-»deutsches* Substrat abzielendes Subjekt zur Debatte. Das ist natürlich nicht der Fall, und Conze konnte das auch wissen, denn er zitiert nur die obenstehenden Worte, aber nicht den klaren Kontext, aus dem hervorgeht, daß auch

6 Am Begriff Volk « ist der Bruch, den die Französische Revolution darstellt, exemplarisch nachvollziebar. Die demokratischen Konnotationen des Begriffs $*$ Volk« treten erst nach 1789 in den Vordergrund, vgl. Grimm, Deutsches Wörterbuch: sinfolge der hier notwendigerweise entstehenden Kämpfe erhielt 'Volk' den Klang eines politischen Parteiworts; es umschloß gewissermaßen die Forderung der Demokratie, die Vertreter einer starken Monarchie liebten es nicht und verwandten für die Gesamtheil der \$taatsbürger lieber das Wort 'Nation', während 'Volk' für die Opposition einen berauschenden Klang hatte (\$.468). In den mittelalterlichen Quellen hat populus/Volk ständischrechtliche bzw. politische Konnotationen und meint immer eine Minderheit im Gegensatz zum sozial-schichtenspezifischen vulgus/Pöbel, womit der *große Haufe« bzw. der *gemeine Mann« bezeichnet wird.

7 Belege wie in dem riche und sunderlich in deutschen landen $(1442$, ebd., S.26) dokumentieren gerade nicht ein »deutsches Selbstbewußtsein«, wie Conze sagt, sondern textgetreu gelesen - die Koexistenz von winternationalem Reich und sdeutschen (d.h. deutschsprachigen) Ländem«, also Sachsen, Baiern, Franken etc. sowie die Nichtexistenz von $*$ Deutschen und $*$ Deutschland $\%$. 
1555 »deutsche Nation* nur auf die Titulatur des Reichsverbands abhebt, nicht auf eine nationale Abgrenzung, einen nationalen Staat im heutigen Sinne: *Darauf Wir uns Gott dem Allmächtigen zu Lob und zu Ehren und Jhr Liebd. und Kayserlicher Majestät zu freundlichem und brüderlichem Gefallen, auch des gnädigen, milden Willens und Vorhabens des Heil. Reiches Teutscher Nation, Unsers geliebten Vatterlands, Unser und des heiligen Reichs gemeiner Stände und Untertanen Nutz, Wolfahrt, Gedeyen und Aufnehmen zu befördern...*; an anderer Stelle heißt es *im Reich Teutscher Nation*. Und wo ausnahmsweise von der $»$ Teutsch Nation, unser(m) geliebt Vatterland * die Rede ist, liegt die gegen Conze sprechende Pointe genau darin, daß es im zu stiftenden Religionsfrieden darum geht $*$ Zertrennung und Untergang* einzelner Reichsteile $*$ zu verhüten*, also gerade nicht Teile des Reichsverbandes zu modernen, andere Gebiete auschließenden *Nationen* zu machen.8

Überhaupt mußte Conze, dem Wissenschaftler, bewußt sein, was Conze, der Nationale, vergaß: Gens und natio können in mittelalterlichen Texten in einem einzigen Satz Familien, Adelsgeschlechter, regionale Gruppen und supragentile Verbände bezeichnen, woraus nüchterne Mediaevisten schon seit geraumer Zeit den notwendigen Schluß zu ziehen, $*$ daß von diesen gentes kein gerader Weg zu den europäischen Nationen führt* (Beumann 1986, S.30).

\section{Das angeblich nationalstaatliche Jahrhundert}

Nationen im modernen Sinne, Nationalismen und Nationalstaaten sind also eine europäische Erfindung - und eine späte obendrein. Sie entstehen, grob gesagt, erst mit der Zerstörung der agrarisch-lokal bestimmten Gesellschaften im Zuge der ökonomischen Modernisierung und Industrialisierung. *Der Tatbestand, eine Nation(alitåt) zu besitzen, ist kein inhärentes Attribut der Menschlichkeit, aber er hat diesen Anschein erworben*

8 Abschied des Augsburger Reichstages (25.9.1555), abgedr. in Zeumer (1913, 5.342, 344). - Im Abschnitt über die *Policey-Ordnung (ebd., S.365f) wird u.a. das Verbot der Einfuhr von $*$ fremder Nation Tücher ins $*$ Heil. Reich Teutscher Nationk begründet. Dabei steht für den ausführlichen Reichstitel gelegentlich die Abkürzung $*$ Teutsche Nation «, aber aus dem Kontext wird unzweifelhaf klar, daß das ganze Reich gleichsam als Zollgebiet gemeint ist. Im umfangreichen Reformwerk gibt es einzige

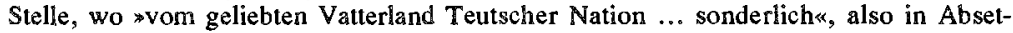
zung vom *Heiligen Reich» gesprochen wird, was man als exklusiven Bezug auf jene oberdeutschen Teile im ostfränkischen Reich interpretieren könnte, wo der religiös motivierte Bürgerkrieg am verheerendsten tobte (ebd., S.342). Freilich ist dies eine sehr wacklige Basis für das laute nationale Capriccio Conzes, denn rund 20 eindeutige Belege im gleichen Text passen überhaupt nicht zur angeschlagenen Melodie. 
(Ernest Gellner). Es handelt sich, bei der meistens staatlich mitgestalteten und mitgetragenen nationalen Territorialisierung im Augenblick des realen Substanzverlusts der Differenz zwischen Menschen unterschiedlicher Herkunft um eine Form von minvented traditions* (Eric J. Hobsbawm): Hymnen, Feste, Folklore, Fahnen, Kasernendrill, Erziehungsrituale - Instrumente im nationalen Baukasten. Im großen Schatten des Nationalen lassen sich sehr vielseitige Geschäfte abwickeln. Der Verdacht, daß hier in der Regel ziemlich Trübes geschieht, wenn man richtig hinsieht, ist für eine aufklärerische und kritische Position unverzichtbar. ${ }^{9}$

Im Falle Deutschlands sind die teutonisierenden Konstruktionen geradezu grotesk: als staatsrechtlich relevanter Begriff taucht $*$ Deutschland* überhaupt erst im 19. Jahrhundert auf - in der Deutschen Bundesakte vom 8.7.1815. Vorher ist die vermeintliche Nation zwischen dem 16. und 19. hauptsächlich adjektivisch und in den Köpfen einiger Intellektueller vorhanden. Zu den objektiven Determinanten des wirtschaftlichen, gesellschaftlichen, kulturellen und - nur ansatzweise - staatlichen Zusammenlebens der Menschen zwischen Mittelalter und 18. Jahrhundert konnte die Nationalität schon allein deshalb keine wichtige Rolle spielen, weil diese Gesellschaften und Staaten, die ihre Truppen oft und gerne aus $*$ Ausiändern* rekrutierten, um den eigenen Bevölkerungsbestand $\mathrm{zu}$ schonen, in

9 Der Philosoph R.Bubner (1991) bejaht die Frage: $»$ Brauchen wir einen Begriff Nation und versucht obendrein in hegelianischer Manier, die Notwendigkeit eines Begriffs der Nation« (gegen die Realitäten und um so schlimmer für diese?) darzutun. Das gelingt ihm nur mittels einer ebenso grotesken wie unhistorischen Gleichsetzung von Staat und Nation sowie einem argumentativen Webfehler, den seine eigene Branche petitio principii nennt - nämlich etwas $*$ schlichtweg zu unterstellen, um dessen Gültigkeit es gerade geht $\ll$. Bubners großspurige Behauptung ( $\$$ Der Nationalstaat ist eine bestimmte Allgemeinheit in Gestalt konstitutionell begründeter Rechtsordnung, die sich mit dem besonderen Profil einer auf Grund ihrer Geschichte unterscheidbaren Nation vermittelta) zerschellt an den tatsächlichen historischen Formationsprozessen, und die vermeintlich nationale $*$ Rechtsordnung ... vermittelt" sich historisch und in der Gegenwart (z.B. in der ehemaligen Sowjetunion oder im untergehenden Jugosiawien) vornehmlich mit dem Charme einer wilden Metzelei im Schlachthof. Dem hilflosen Versuch, das *historisch greifbare Selbst « als Substrat von *Selbstbestimmung « ausgerechnet im Konstrukt Nation anzusiedeln, ergeht es wie dem whistorischen Recht $\%$ : der regressus ad infinitum ist so unvermeidlich wie der salto mortale in die Opernwelt geschichtsphilosophischer Spekulation. Solchen Begriffsakrobaten wurde schon vor über 100 Jahren heimgeleuchtet; an einem historischen Wendepunkt schrieb. E.Renan an D.Strauß am 15.9.1871: $\times$ Lothringen hat einmal zum deutschen Reich gehört, darüber besteht kein Zweifel... Fast überall da, wo die hitzigen deutschen Patrioten sich auf ein altes germanisches Recht berufen, können wir ein noch älieres keltisches belegen, und vor den Kelten lebten dort, wie man sagt, die Allophylen, die Finnen, die Lappen; und vor den Lappen waren es die Höhlenmenschen und vor den Höhlenmenschen die Orang-Utans. Für eine solche Geschichtsphilosophie gibt es als ein dingliches Recht in der Welt nur das Recht der Orang-Utans, die ungerechterweise von der bösen Zivilisation vertrieben worden sind." (Rougemont 1962, S.282) 
einem strikten Sinne a-national waren. Die Oberschichten, allen voran Adel und Klerus, orientierten sich an gesamteuropäisch generierten Handlungskodizes, Kulturmustern und Moden; oft sprachen sie die Sprache »ihres* Volkes nicht oder nur unzureichend. Für das Volk, für die Unterschichten also, hörte die Welt buchstäblich am Dorfrand auf. Sie waren nach Sprache, Sitten, Kultur, sozialen Beziehungen und wirtschaftlichen Verflechtungen während Jahrhunderten lokal, allenfalls regional geprägt. Und die Angehörigen der sehr dünnen Mittelschicht, die allein »Nationalität« als Abgrenzungs- und Anerkennungsmerkmal hätte ausbilden können, verkehrten untereinander als Gelehrte lateinisch und als Händler kommerziell - auf jeden Fall eher nach kosmopolitisch-universalistischen Standards als nach nationalen.

Auch die Sprache bildet - entgegen dem naiven, national getrimmten Denken des 19. Jahrhunderts - dort kein objektives Abgrenzungskriterium mehr, wo Mehrsprachigkeit die Norm abgibt und es mithin nur vom Willen der Individuen oder den mehr oder weniger zufälligen Einflüsterungen, denen sie ausgesetzt sind, abhängt, welcher (Sprach-) Nation sie angehören wollen. Als Mitglied der polnisch-deutsch-litauisch wversippten* Oberschicht z.B. konnte man sich sprachlich, kulturell oder politisch je nach Opportunität an mindestens drei Nationalitäten positiv orientieren - an der polnischen, der deutschen oder der litauischen; und negativ besetzt blieben wenigstens zwei, die jüdische und die russische; für die östlichen Teile der ehemaligen Habsburger Monarchie gilt Ähnliches, ebenso wie für große Bereiche des Balkans. Der US-Soziologe W.B. Pittsburg stellte schon 1882 fest: »Der einzige Weg, um zu entscheiden, ob ein Individuum $\mathrm{zu}$ einer oder der anderen Nation gehört, ist, es zu fragen*.

Im angeblich nationalstaatlich dominierten 19. Jahrhundert gab es genau einen Staat, den man ohne größere Vorbehalte als homogenen Nationalstaat bezeichnen könnte: Portugal (Hobsbawm 1991, S.112).10 Richtig ist freilich, daß es sich zum Teil um ein von nationalistischen Ideologien beherrschtes Jahrhundert gehandelt hat, was notorisch verwechselt und zusammengeworfen wird.11 Der Nationalstaat war vor allem der ideologische Panzer, den sich (bis 1914 fast auschließlich) jene mittleren sozialen Klassen und Schichten gegen die Bedrohung überzogen, die sie als die gefährlichste am Horizont aufgehen sahen: die internationalistische und sozialistische Arbeiterbewegung. Den Höhepunkt erreichte der Nationalis-

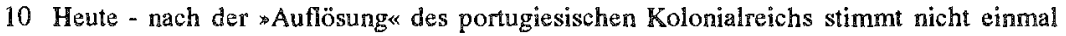
mehr das, wovon man sich auf den Straßen Lissabons schnell überzeugen kann.

11 "Die grundsätzliche Loyalität des neuen Nationalismus galt paradoxerweise nicht 'dem Land', sondern allein seiner Auffassung von diesem Land - einer ideologischen Konstruktionk (Hobsbawm 1991, S.111). 
mus jedoch nicht im 19., sondern erst im 20. Jahrhundert - nach dem epochalen Bruch von 1917, d.h. dem Eintritt der USA in den bislang europäischen Krieg und dem Ausbruch der Oktoberrevolution. Der Nationalismus und der national definierte Staat bot jenen mittleren sozialen Klassen und Schichten vor allem in der zerfallenen Donaumonarchie einen Schutz gegen die drohende soziale Revolution; den siegreichen Alliierten erschien die Zerschlagung des Habsburger Imperiums in Kleinstaaten als probates Mittel, das kriegerische Potential aufzusplittern (vgl. ebd., 153f.) und zu »neutralisieren $*$. Wenn die Gelehrten-Floskel vom Pyrrhussieg je zutraf, dann auf die Geschichte und die Praxis des 1918 durch die Sieger proklamierten Prinzips des »nationalen Selbstbestimmungsrechts»。

\section{5. "Nationales Selbstbestimmungsrecht" nach 1918}

Historiker, Juristen, Philosophen, Politiker und Lexikographen sprachen im 19. Jahrhundert quer durch das politische Spektrum von den halbfeudalen Konservativen bis zu den Demokraten nicht vom »nationalen Selbstbestimmungsrecht * oder vom *Selbstbestimmungsrecht der Völker * wie die UNO-Resolution Nr. 545 vom 5.2.1952, sondern vom Nationalitätenprinzip* (Bluntschli, Brater 1862, S.152ff). 12 Sie wußten warum. Wissenschaft und Politik hatten im 19. Jahrhundert eine durchaus realistische Vorstellung davon, was passieren kann, wenn man Individuen, Gruppen oder gar ganzen Völkern ein "Selbstbestimmungsrecht* einräumt. Im fachphilosophischen Jargon mochte der Kantische Begriff angehen, in der Politik hing ihm der Ludergeruch der *classes dangereuses * an. Außerhalb der gerade nicht national kostümierten Theorien des Anarchismus und des Sozialismus Marxscher Prägung hatten Begriffe wie "Selbstbestimmung", "Emanzipation* und "Autonomie* deshalb geringe Bedeutung und keinerlei gesichertes theoretisches oder politisches Heimatrecht (von ihrer Eignung für nationalistische Rituale träumte noch nicht einmal jemand).

Woodrow Wilson, der das Prinzip »nationaler Selbstbestimmung* mit seinem Friedensprogramm vom 8.1.1918 in die internationale Debatte lancierte, mußte sich von seinem engsten Berater, dem Staatssekretär Robert

12 Im Prinzip gilt: $\rightarrow$ Jede Nation ist berechtigt, sich zum Staate zu einigen und zu organisieren“ (ebd. S.157). Freilich nur unter der Bedingung, *die historische Rechtsordnung .. zu respektieren«, (ebd., S.159). SchlieBlich: $\gg$ Die höchste Staatenbildung beschränkt sich nicht auf eine einzelne Nationalität, sondern verbindet verschiedene nationale Elemente zu einer gemeinsamen menschlichen Ordnung*. Einmal mehr: das national verblendete und verbiesterte Jahrhundert ist nicht das letzte, sondern das laufende. 
Lansing vorrechnen lassen, welches Chaos die Floskel anrichten konnte unabhängig davon, ob sie wörtlich oder nur thetorisch verstanden wurde: »Das ganze Wort 'Selbstbestimmung' ist mit Dynamit bis zum Rande geladen. Es wird Hoffnungen erwecken, die sich nimmer erfüllen lassen. Ich fürchte, daß es tausende und abertausende Leben kosten wird... Welch ein Verhängnis, daß dies Wort je geprägt wurde! Welch Elend wird es über die Menschen bringen! « (Lansing 1921, S.73). Zwar gilt das zwangsläufig nur, wenn man sich Selbstbestimmung als »national e eingefärbte und national überhaupt definierbare 13 vorstellt - aber davon wollte die ebenso staatsfixierte wie bürgerliche Welt nichts wissen, die mit der Parole den mittelständischen Nationalismus auf dem Balkan zufriedenstellen, gleichzeitig den expansiven Nationalismus in Deutschland bändigen und obendrein den revolutionären Vielvölkerstaat Sowjetunion konterkarieren wollte.

Auf dem Balkan brach das Prinzip "nationale Selbstbestimmung* nicht einfach ein. Es stürzte einen halben Kontinent ins voraussehbare Elend. Die Versuche, im Osten und Südosten Europas nach 1918, das Kriterium der Nationalität zur Staatsbildung heranzuziehen, mußte scheitern, weil in diesen Räumen seit Jahrhunderten ethnisch, kulturell und sprachlich vielfach durchmischte Völker lebten und leben. Soziale Bindungen, ökonomische Strukturen und politische Beziehungen folgten bis dahin in diesen Regionen entlang vielerlei Traditionen und Prinzipien - nur nicht dem nationalen. Das vollkommen willkürliche nationale Prinzip erzeugte höchst bunte "National «staaten, die mit ihren Minderheiten keineswegs humaner verfuhren als die alte Herrschaft. Außerdem schuf der kleinstaatliche Nationalismus nun jede Menge *national * begründeter $\gg$ Anschluß-* bzw. *Befreiungs*-Bewegungen. Mit der Unterwerfung des vielfältigen lokalen und regionalen Zusammenlebens unter den Primat des Nationalen und Nationalstaatlichen wurden die Konflikte auf dem Balkan dramatischer und unlösbarer als je zuvor. Die übergestülpten nationalen Ord-nungsrezepte satter $\gg$ Nationalstaats*-Realisten bereiteten dem nationalistischen Populismus und der vulgär-völkischen Demagogie den Weg als Herrschaftsinstrumente.

In den 20 er Jahren gab es eine von den Regierungen in Osteuropa hoch-

13 Alle drei Komponenten des Pseudobegriffs mnationales Selbstbestimmungsrecht» werden mindestens doppel- bzw. mehrdeutig, wenn das Selbst, das angeblich bestimmt, gleichzeitig national und rechtlich verfaßt sein soll. Wird die Nation streng definiert, gerät universalistisches Recht zur fictio iuris innerhalb willkürlicher wethnischer Grenzen; hält man sich an Recht, das diesen Namen verdient, muß man alle Hoffnung auf Fremdes ausgrenzenden Nationalitätenzauber ebenso fahren lassen wie Identitälsstiftung durch rechtliche Privilegierung einer Nationalität - z,B. im Wahl- und Beamtenrecht und anderswo. 
bezahlte Riege von Fachleuten, die sich mit nichts anderem befaßten als mit $»$ Methoden der Nationalitätenzählung in Österreich-Ungarn und in der Tschechoslowakeik. Das war das akademische Lösungsmuster für die die hausgemachten Nationalitätenkonflikte. Subkutan wurstelten nationalistisch angeheizte Terroristen ganz unakademisch im Namen »nationaler* Selbstbestimmung mit Dynamit und Brownings. Für beide - Nationalitäten-Zähler und Nationalitäten-Dynamiter - gab es viel zu tun, denn die Nachhilfe für jede $*$ Nation* - ob mittels Fragebögen oder Anschlägen rief allemal eine oder mehrere Gegen-Nationen auf den Plan. Obendrein rutschten die längst überforderten staatlichen Organe in die Konflikte hinein oder installierten sich als diktatorische Schiedsrichter oberhalb *ihrer* Nationalitäten. Der Zerfall der Staaten war in beiden Fällen absehbar.

$\mathrm{Da}$ es niemals ein allgemein akzeptiertes und akzeptables Verfahren für die Bestimmung dessen gab, was eine »Nation« ist, geschweige denn eine konsensfähige Theorie und Praxis, wie und von wem das $»$ Selbstbestim-

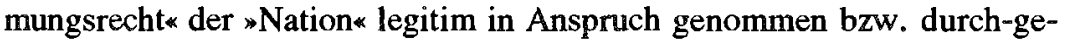
setzt werden soll, lief dieses famose Recht schnurstracks auf eine Gleichsetzung von Recht und Gewalt hinaus.

Das »nationale Selbstbestimmungsrecht* ist historisch betrachtet nicht weniger fragwürdig als die Passierscheine, die sich die Convention nationale im Namen der Nation française am 19.11.1792 und am 15.12.1792 ausstellte, um den unterdrückten Völkern - ohne sie zu fragen - "Brüderlichkeit und Hilfe" (Forrest 1988, S.146ff) anzudienen für die Befreiung von despotischer Herrschaft. Als Exportartikel verkam »nationale Selbstbestimmung* im ersten, napoleonischen Anlauf schnell zur »brüderlichen Hilfe* im Namen der *nationalen Befreiung*, 14

14 Kein Mißverständnis westeuropäischer und amerikanischer Linker ist gravierender und langlebiger, als die Subsumierung der Befreiung von Völkerschaften aus kolonialer Abhängigkeit unter dem unpassenden Terminus $*$ nationale Befreiung«. Erstens handelte es sich bei den abhängigen Völkern in den wenigsten Fällen um eine *Nation oder auch mehrere wationen sozialrevolutionärer Befreiungsbewegungen meistens nur eine Not zur Tugend: weil die soziale Revolution ganz oder auf halbem Wege stecken blieb, bedurfte sie eines nationalen Kostüms, um wenigstens Reste von Massenloyalität und Legitimität zu behalten. Der algerische wie der kubanische Nationalismus sind typisch für diese Deformation. Drittens konnte der Kampf gegen Imperialismus und Kolonialismus nur deshalb den Schein eines »nationalen Kampfes« annehmen, weil führende Köpfe der Befreiungsbewegungen europäische Theorien, Begriffe und Traditionen umstandslos auf Afrika, Asien und Lateinamerika übertrugen. Ob historische Ignoranz, Befangenheit in leninistischen Schematismen oder schlichte terminologische Schlamperei dafür verantwortlich ist, wäre am Einzelfall zu klären. 


\section{6. "Nationale Selbstbestimmung " heute}

Roosevelts und Churchills Atlantik-Charta vom 14.8.1941 enthielt eine unverbindliche Formulierung des nationalen Selbstbestimmungsrechts. Die UNO-Charta vom 24.10.1945 anerkennt den Grundsatz der Selbstbestimmung im allgemeinen, aber erst die Resolution Nr. 545 vom 5.2. 1952 statuiert explizit ein $»$ Selbstbestimmungsrecht der Völker* bzw. $»$ das Recht der Völker und Nationen auf Selbstbestimmung* (Resolutionen 1990, \$.32) - freilich ohne ein Wort der begrifflichen Klärung. Weder *Volk*, *Nation* oder $*$ Land* noch Inhalt und Umfang des Rechts auf "nationale Selbstbestimmung oder jenes auf "nationalek Unabhängigkeit werden genauer gefaßt in den nachfolgenden Resolutionen Nr. 1514 vom 14.12.1960 über $\rtimes$ die Gewährung der Unabhångigkeit an koloniale Länder und Völker*, Nr. 2649 vom 30.11.1970 über die »weltweite Verwirklichung des Rechts der Völker auf Selbstbestimmung* und Nr.40/56 vom 2.12.1985 zum $» 25$.Jahrestag der Deklaration über die Gewährung der Unabhängigkeit an koloniale Länder und Völker (ebd. S.44ff, 72ff, 88ff). Ein Grund dafür liegt im prinzipiellen Opportunismus staatlicher Eliten und politischer Klassen, demzufolge zwar jeder "nationale Staat* jeden anderen anerkennt, freilich mit dem notorischen Vorbehalt, aus Gründen "nationaler Selbsterhaltung* (die immer einseitig festgestellt werden) den hehren Grundsat $z$ desavouieren zu dürfen.

Was speziell die nationalstaatliche Unabhängigkeit « betrifft, so ist sie weitgehend schimårisch, wenn man sieht, wieviele Staaten - zumal europäische - in internationale bzw. zwischenstaatliche Strukturen, Bündnisse, Gemeinschaften etc. eingebunden sind und viele Kompetenzen abgetreten haben. Ganz zu schweigen von der internationalen wirtschaftlichen und kapitalistischen Verflechtung unter der Dominanz von ein paar Dutzend weltweit operierenden Großkonzernen, die jeder »nationalen« Regierung mehr oder weniger direkt diktieren können, was anliegt. 15 Unabhängig sind fast alle Staaten nur noch bei Unwesentlichem; in den Kernbereichen regieren international strukturierte kapitalistische Abhångigkeitsverhältnisse vielfåltiger Art und Strenge - trotz aller nationalen Rhetorik und militärischen Folklore.

Zwar bietet die (fingierte) nationalstaatliche Territorialisierung keinerlei Gewähr dafür, daß reale politische, wirtschaftliche und soziale Probleme gelöst werden. Im Gegenteil: in der Regel verschärfen solche anachroni-

15 Wenn die Schweiz ein amerikanisches Kampfflugzeug beschaffen will, bestehen die Waffenproduzenten auf $50 \mathrm{Mio}$ sFr. Anzahlung, noch bevor das zuständige Parlament die Beschaffungsvorlage zu Gesicht bekommt. Eine nette Lektion, die wohl ein tough guy oder ein tough baby aus der parlamentarischen Grundweisheit, wonach immer $B$ sagt, wer A gesagt hat, gelemt hat. 
stischen Territorialisierungen bestehende Konflikte und erzeugen zusätzliche. Das gilt für die Auflösung der ehemaligen Sowjetunion ebenso wie für die Verhältnisse im untergehenden Jugoslawien. "In einer Welt, in der wahrscheinlich nicht mehr als ein Dutzend von insgesamt 170 Staaten überzeugend von sich behaupten können, daß ihre Bürger nicht nur in einem abstrakten Sinne mit der Gesamtheit einer einzelnen ethnischen oder Sprachgruppe identisch sind - in einer solchen Welt ist jeder Nationalismus, der sich auf die Herstellung einer solchen Homogenität gründet, nicht nur nicht wůnschenswert, sondern selbstzerstörerisch" (Hobsbawm 1991, 214). Die Berichte und Bilder von den Tatorten belegen dies täglich.

Der konservative Historiker Hans Rothfels, der vor dem Krieg in Königsberg lehrte und 1933 emigrieren mußte, sprach 1952 von der »offenbaren Absurdität, die dem Ideal des autonomen Nationalstaats angesichts der Dimensionen des Zeitgeschehens anhaftet (Rothfels, 1952, 124). Seither haben sich die sozialen, wirtschaftlichen und politischen Verhältnisse so verändert, daß $\gg$ Absurdität« geradezu schönfärberisch klingt. Arbeitsemigration, europäische Integration und starke Flüchtlingsbewegungen haben alle europäischen Gesellschaften und Staaten zu ausgesprochenen Vielvölkerstaaten gemacht. Überall hinkt demgegenüber die nationalstaatliche Ideologie und Praxis, mit der je nach Land 10 bis $30 \%$ der Einwohner vom Wahlrecht und anderen Rechten ausgeschlossen werden, den gesellschaftlichen Realitäten hinterher. Besonders grotesk ist die Lage der BRD, wo die politische Klasse mit dem Beischluß der ex-DDR nach Grundgesetz Art. 23 absichtlich den Weg einer Filialeneröffnung unter pseudo-nationalstaatlichem Vorzeichen wählte und nicht denjenigen einer demokratisch legitimierten Neukonstititution nach Art. 146 (unter Einbeziehung aller dauerhaft im Lande wohnenden und arbeitenden Menschen). Die politische Klasse erweckte mit ihrem Vorgehen im nationalistischen Milieu - hüben und vor allem drüben - Hoffnungen auf eine Fortsetzung und Steigerung dieses Kurses bis hin zu *richtiger* nationaler Politik; sie schürte nationale Hoffnungen, die sie natürlich nicht erfüllen kann. Wenn die Bonner Politik den Rechtsradikalismus auch nicht erzeugte, so trieb sie ihm doch viele Menschen zu. Gleichzeitig frustierte die kabinettspolitisch legitimierte Erweiterung im nationalen Galopp nicht nur viele Deutsche, sondern alle im Land lebenden Ausländer, die eine demokratische Gleichberechtigung anstreben. Auslånder und Flüchtlinge kriegen die Ressentiments des nationalen Mobs, den mittlerweile die finanziellen Kosten der Vereinigung mehr drücken als ihn die nationale Einheit stimuliert, am direktesten zu spüren. 


\section{Literatur}

Beumann, H. (1986): Zur Nationenbildung im Mittelalter, in: Nationalismus in vorindustrieller Zeit, hg.v. O.Dann, München.

Bluntschli, J.C.; Brater, K.L. (1862): Deutsches Staatswörterbuch, Bd. 7, Artikel Nation und Volk, Nationalitätenprinzip, Stuttgart, Leipzig.

Bubner, R. (1991): Staat - und Weltstaat, NZZ v. 5.7.1991.

Busche, J. (1991): Des Deutschen Vaterland, in: Kursbuch 104

Conze, W. (1983): "Deutschland« und *deutsche Nation« als historische Begriffe, in: Die Rolle der Nation in der deutschen Geschichte und Gegenwart, hg.v. O.Büsch u. J.J.Sheehan, Berlin 1985.

Forrest, A. (1988): Art. La révolution et l'Europe, Dictionnaire de la révolution française, éd. F.Furet, M.Ozouf Paris.

Graus, F. (1969): Böhmen zwischen Bayern und Sachsen. Zur böhmischen Kirchengeschichte des 10.Jh., Historica 17.

Grimm, J.u.W. (1951): Deutsches Wörterbuch, Bd. 12/1.

Guenée, B. (1971): L'occident aux XIVe et XVe siècles. Les États, Paris.

Hobsbawm, E.J. (1991): Nationen und Nationalismus. Mythos und Realität seit 1780, Frankfurt.

Lansing, R. (1921): Die Versailler Friedensverhandlungen, Berlin.

Resolutionen zum Selbstbestimmungsrecht der Völker (1990), hg.v. J.Schulz., K.Mann (= Die Vereinigten Nationen und ihre Spezialorganisationen. Dokumente, Bd. 3, T1. 2 , Berlin)。

Rothfels, H. (1952): Zur Krise des Nationalstaats, in: ders., Zeitgeschichtliche Betrachtungen, Göttingen 1959.

Rougemont, D.de (1962): Europa. Vom Mythos zur Wirklichkeit München.

Schlesinger, W. (1965): Die Auflösung des Kaiserreiches, in: Ausgewählte Aufsätze 1965. 1979, Sigmaringen 1987.

See, K.v. (1970): Deuische Germanesideologie, Frankfurt.

Sieyes, E.J. (1789): Was ist der dritte Stand? in: ders., Politische Schriften, hg.v. E.Schmitt u. R.Reichhardt, Darmstadt, Neuwied 1975.

Taine, H. (1893): Les origines de la France contemporaine, Tl. 3, Bd. 3, Paris 1921.

Vossler, K. (1913): Frankreichs Kultur und Sprache. Geschichte der französischen Schriftsprache von den Anfängen bis zur Gegenwart, Heidelberg 1929.

Wenskus, R. (1961): Stammesbildung und Verfassung. Das Werden der frïhmittelalterlichen gentes, Köln, Graz.

Werner, K.F. (1984): Histoire de France, t. 1: Les origines, Paris.

Werner, K.F. (1986): Artikel $\gg$ Deutschland «, in: Lexikon des Mittelalters, Bd. 3, München, Zürich

Werner, K.F. (1992): Artikel Volk, Nation, in: Lexikon Geschichliche Grundbegriffe, hg.v. O.Brunner, W.Conze, R.Koselleck, Bd. 7 (im Druck).

Zeumer, K. (1913): Quellensammlung zur Geschichte der Deutschen Reichsverfassung in Mittelalter und Neuzeit, hg.v. K.Zeumer Tübingen 